\title{
Feasibility of impact-acoustic emissions for detection of damaged wheat kernels
}

\author{
Tom C. Pearson ${ }^{\mathrm{a}, *}$, A. Enis Cetin ${ }^{\mathrm{b}}$, Ahmed H. Tewfik ${ }^{\mathrm{c}}$, \\ Ron P. Haff ${ }^{\mathrm{d}}$ \\ ${ }^{\text {a } U S D A-A R S, ~ M a n h a t t a n, ~ K S, ~ U S A ~}$ \\ b Bilkent University, Ankara, Turkey \\ ${ }^{\mathrm{c}}$ University of Minnesota, Minneapolis, MN, USA \\ d USDA-ARS, Albany, CA, USA \\ Available online 2 September 2005
}

\begin{abstract}
A non-destructive, real time device was developed to detect insect damage, sprout damage, and scab damage in kernels of wheat. Kernels are impacted onto a steel plate and the resulting acoustic signal analyzed to detect damage. The acoustic signal was processed using four different methods: modeling of the signal in the time-domain, computing time-domain signal variances and maximums in short-time windows, analysis of the frequency spectrum magnitudes, and analysis of a derivative spectrum. Features were used as inputs to a stepwise discriminant analysis routine, which selected a small subset of features for accurate classification using a neural network. For a network presented with only insect damaged kernels (IDK) with exit holes and undamaged kernels, $87 \%$ of the former and $98 \%$ of the latter were correctly classified. It was also possible to distinguish undamaged, IDK, sprout-damaged, and scab-damaged kernels.
\end{abstract}

(c) 2005 Elsevier Inc. All rights reserved.

Keywords: Neural network; Spectral analysis; Insect damage kernels; Sorting; Acoustic emissions

\footnotetext{
* Corresponding author. Fax: +785 5375550.

E-mail address: tpearson@gmprc.ksu.edu (T.C. Pearson).
} 


\section{Introduction}

In most years, many types of wheat kernel damage occur in limited quantities from isolated growing areas. Three common types of damage that impact flour quality and/or safety are fungal damage, sprout damage, and insect damage. Several fungi can infect kernels before and after harvest. The most important of these is Fusarium graminearum, which creates "scab" damage and may lead to toxins known to cause cancer [1]. Scabdamaged kernels generally have a white, chalky appearance covering most of the exterior surface [2]. Sprout damage occurs when the moisture content at harvest time is too high, causing germination of the seed. The result is a sprout shoot emerging from the kernel and/or a discolored germ. Enzymes which convert starch to sugar are activated during sprouting and can impact bread quality if present in the flour. Internal insect infestation degrades the quality and value of wheat and is one of the most difficult defects to detect. Infestation causes grain loss by consumption, contaminates the grain with excrement and fragments, causes nutritional losses, and degrades end-use quality of flour [3]. Levels of insect infestation are therefore a major factor in the grading of wheat quality. USDA standards allow up to 31 insect damaged kernels (IDK) per 100 grams (about 3000 kernels) of wheat for grades U.S. No. 1-U.S. No. 5 [4]. Samples with more than 31 instances of IDK are graded as U.S. sample grade. Food and Drug Administration standards consider wheat infested at the same limit of 32 or more instances of IDK [5]. However, wheat millers usually specify that wheat loads must contain less than five IDK per $100 \mathrm{~g}$. Inspecting for IDK is labor intensive and may overlook most of the infested kernels where an immature insect has not emerged from the kernel.

Wheat kernels become infested when an adult female bores a small hole (roughly $0.05 \mathrm{~mm}$ ) into the kernel, deposits an egg, and seals the hole with a mucilaginous plug. The plug is the same color as the wheat surface, making it difficult to detect by external examination. When the egg hatches, the larvae develops and feeds, forming tunnels inside the kernel. Upon maturity, the insect exits the kernel, leaving an exit hole. Inspectors counting IDK in a sample look for this exit hole. However, kernels which contribute the most to reduced flour quality are those containing immature insects, which are generally not detected by inspectors. These kernels containing hidden infestations are not part of the IDK classification. Not only do these insects mature and lay more eggs, allowing the infestation to spread, but also contribute to the insect fragments in the flour.

Sprout, scab, and IDK are identified in the grading process by manual inspection of small samples of grain. However, normal procedures for handling and transporting lead to mixing of damaged with undamaged kernels. Hence, the ratio between the two is generally small, requiring large samples for damage to be accurately quantified. To date, rapid and economical methods to quantify IDK and hidden insect infestations in wheat samples have not been developed. The need for rapid inspection of individual kernels makes development of such a method problematic. Because the occurrence of IDK and hidden infestations is very small as a fraction of the total number of kernels to be evaluated, a low false-positive error rate (error in classifying good kernels as damaged) is essential to avoid discarding large amounts of good product. A variety of methods have been attempted to detect internal insect damage, and others are under development. These include cracking and flotation [6], X-ray imaging [7-9], acoustic detection of larval movement and chewing 


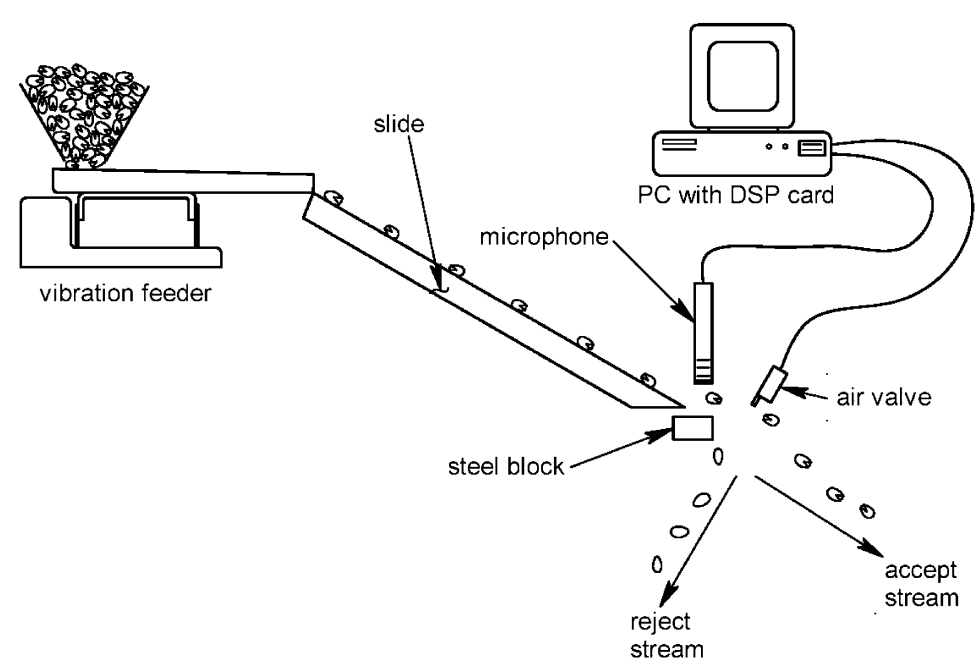

Fig. 1. Schematic of pistachio sorter based on acoustic emissions.

[10-13], carbon dioxide measurement [14], and immunological methods [15]. However, most of these methods have only achieved limited implementation either because they are slow, labor intensive, expensive, and can only detect specific insect species, or they cannot quantitatively measure insect infestation levels. More recently, an image-analysis program has been developed to automatically scan X-ray images for insect infestation [7]. Other researchers have investigated use of NIR spectroscopy to detect hidden insects in wheat kernels $[16,17]$. Finally, a digital signal-processing method has been developed to work in conjunction with a Perten single-kernel characterization system (SKCS) to detect insectdamaged kernels [18]. This method further processes the crush force signal produced by the SKCS as it crushes wheat kernels. As expected, insect damaged kernels produce different crush force signals than un-damaged kernels. X-ray, NIR methods, and SKCS methods suffer from high false-positive error rates and are cost prohibitive for many commercial applications. Thus, no economically viable equipment utilizing these technologies has become available for detection of internal insects.

Sprout, scab, and insect damage may be detectable by impact acoustic emissions since the physical and/or structural properties of these kernels are different compared with undamaged kernels. Sprouting causes conversion of starch to sugars, and emergence of the shoot is accompanied by splitting of the kernel. Fungi infecting scab-damaged kernels can make the endosperm porous and the kernel shrink and lose weight [19]. Finally, the insect tunnels alter the structure of infested kernels. Impact-acoustic emissions has been successfully used as the basis for a device that separates pistachio nuts with closed shells from those with split shells [20-22]. In that system, shown in Fig. 1, pistachio nuts impact a steel plate and the resulting acoustic emissions are analyzed in real time. When a closed shell is detected, an air valve is activated and the nut diverted. Classification accuracy is approximately $97 \%$, with a throughput rate near 40 nuts per second. The device is economical (approximately \$5000), reliable in a food processing environment, and requires minimal 
maintenance or expertise. Simple features such as amplitude of the sound and decay rate of the amplitude are extracted from the acoustic signal as a basis for sorting.

\subsection{Non-destructive techniques using acoustic emissions}

Acoustic-emission techniques are widely used to monitor structures and machine parts, especially bearings and gears, to determine if failure is imminent [23]. Common signal processing includes thresholding, counting points above or below a threshold, modeling signals in the time-domain with damped sinusoids, using a combination of techniques, such as covariance analysis, principal component analysis, differential timing analysis and neural networks to detect and classify acoustic emissions in the presence of strong background sound signals [24] and [25], and detection of repetitive pulses [26]. Monitoring of machine parts usually allows capture and analysis of several periods of a repeated signal. For example, certain types of slow-moving bearings emit a "tick" on every rotation when failure is imminent. For sorting of agricultural products in real time on a processing line, there is limited time (a few milliseconds) to acquire and process data, classify the object, and trigger a rejection mechanism.

Historically, the fields of oil exploration and seismic activity due to earthquakes have produced the most extensive research of acoustical signal processing methods. In the case of oil exploration, a charge is detonated and the acoustic signals of echoes at plate boundaries and oil reservoir surfaces are analyzed [27]. Early studies in this field led to the development of the cepstrum, defined as the inverse discrete Fourier transform (DFT) (or inverse discrete cosine transform) of the log DFT of the time domain signal [28]. The cepstrum characterizes variations in the spectrum due to overtones of the underlying fundamental resonating frequency. Portions of the cepstrum are now commonly used as feature vectors for representing human speech and musical signals. Cetin et al. [22] developed an improved classification scheme for the pistachio-sorting device developed by Pearson [20] through use of cepstrum computations. The sound-feature vector, used to distinguish closed from split shells, consists of mel-cepstral coefficients along with principal components of the magnitude of acoustic-impact emission signals. This technique is common in speech-recognition systems [21].

Other acoustical methods have been studied to detect firmness in fruits. Several researchers have found a good correlation between fruit firmness and resonant frequency $[29,30]$. These acoustical systems typically involve tapping the food with a plunger, recording the sound generated, and digitally processing the signal to extract dominant frequency bands or other features correlated with firmness. Younce and Davis [31] developed such a system to measure firmness of cherries using impact acoustics. Sugiyama et al. [32] developed an acoustical firmness tester for melons that measured sound-transmission velocity. This technique eliminated some error caused by size and shape variations among the fruits. While these methods are able to measure certain fruit properties, they are not readily adaptable to high-speed inspection. A more likely candidate for high-speed inspection of wheat would be to capture and analyze the signal generated when the kernels are dropped and impact a steel plate, as in the case of the pistachio-sorting device described above.

The objective of this study was to demonstrate the feasibility of using signal processing algorithms to separate damaged and undamaged wheat kernels impacting a steel plate at 
high speed in real time. Emphasis was given to detecting IDK and hidden infestations, which are among the more difficult types of damage to quantify. The overall goal of the project was to detect, classify, and remove contaminated and damaged kernels in real time using the sound generated when a kernel strikes a steel plate (impact acoustics) as the basis for sorting. This mode of acoustic excitation is easily adapted to high-throughput sorting systems.

\section{Materials and methods}

Rice Weevils [Sitophilus oryzae (L)], a common stored-grain insect, were reared in jars of hard red winter (HRW) wheat. The wheat, grown in central Kansas, Oklahoma, and Colorado in 2001 and 2002 was a mixture of Pioneer 2137, 2163, 2174, Karl and Jagger varieties. Selected kernels were frozen to kill the insects and larvae, held at room temperature for six weeks to allow moisture to equilibrate (10.5\%), then X-rayed on film at an exposure of $18 \mathrm{kV}, 3 \mathrm{~mA}$, for 2 min exposure. Kernels with interior insect damage at the pupal stage were identified by examination of the X-ray films. Pupae were differentiated from less mature larvae by their larger size and the presence of a snout and/or limbs. These images represent hidden insects at the most advanced stage before exiting the kernels. Since the immediate objective of this study was to demonstrate feasibility, less mature infestations were not included. Figure 2 shows a radiograph of undamaged kernels (left), kernels with pupae present (center), and kernels in which the adult insect has exited the kernel (IDK) (right). Wheat from the same source was hydrated for three days to allow germination to begin and cause sprout damage. Scab-damaged HRW kernels of unknown variety were provided by the Federal Grain Inspection Service (FGIS). From the wheat sources described, 900 undamaged kernels were collected along with 500 each of pupae-stage infested kernels, IDK, scab-damaged, and sprout-damaged kernels. This gave a total of 2900 kernels, 2000 damaged and 900 undamaged, to be used for classification experiments.

\subsection{Experimental apparatus}

A schematic of the experimental apparatus for singulating wheat kernels, dropping them onto the impact plate, then collecting the acoustic emissions from the impact is shown in
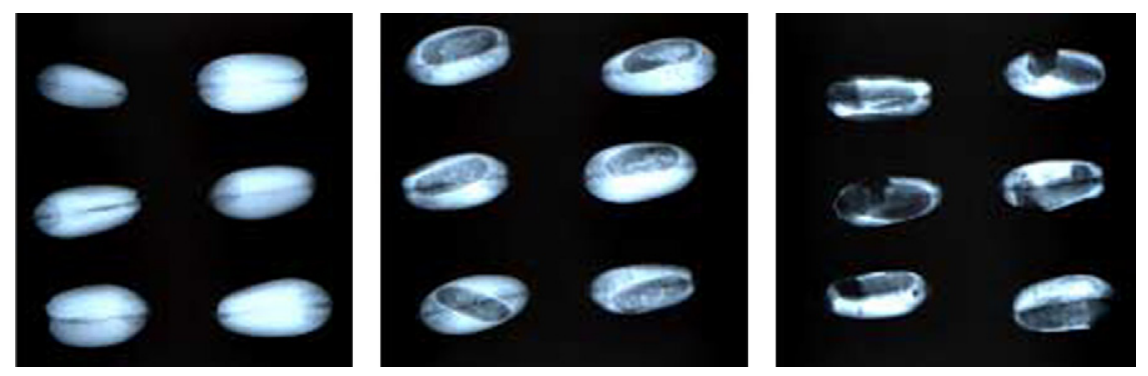

Fig. 2. Radiographs of wheat kernels with different stages of insect damage. From left to right, undamaged kernels, kernels with damage at the pupal stage, and IDK where the insect has matured and exited the kernel. 


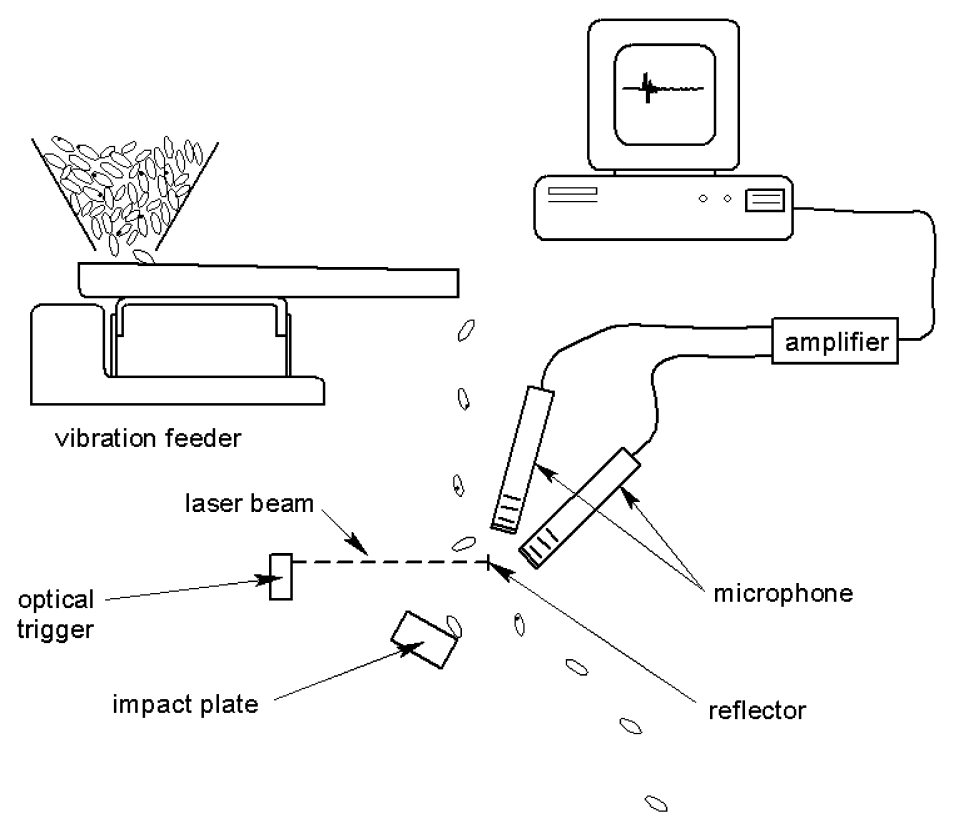

Fig. 3. Schematic of experimental apparatus for collecting acoustic emissions from wheat kernels.

Fig. 3. The bulk kernels were transformed into a single-file stream using a vibration feeder (F-TO-C, FMC-Syntron, Homer City, PA), which consisted of a vibrating bulk hopper and a steel, V-shaped, trough. The impact plate was a polished block of stainless steel approximately $7.5 \times 5.0 \times 10 \mathrm{~cm}$. The mass of a single wheat kernel is negligible compared to the mass of the impact plate, hence the possibility of vibrations from the plate interfering with acoustic emissions from kernels was minimized. The drop distance from the feeder to the impact plate was $40 \mathrm{~cm}$ and the plate was inclined at $30^{\circ}$ above the horizontal. This angle was determined by trial and error. Flatter angles of incline produced a stronger signal but kernels would tend to bounce twice before falling off of the plate. This was not conducive to high speed sorting. The angle of $30^{\circ}$ was found to produce adequate signal and project the kernel off of the plate after the first bounce. Larger drop distances also produce a stronger signal; however, during larger drops, the kernel trajectory would not be as consistent. For example, kernels would fall into a one centimeter diameter spot for a $40 \mathrm{~cm}$ drop but that increased to $3 \mathrm{~cm}$ for an $80 \mathrm{~cm}$ drop. To reliably divert kernels, the location where they drop needs to be consistent so a drop of $40 \mathrm{~cm}$ was selected.

Two microphones (4939 L with 2669 L pre-amp, Bruel and Kjaer North America, Norcross, GA), sensitive to frequencies up to $100 \mathrm{kHz}$, were used to capture audible and ultrasonic acoustic emissions from the impact of the wheat kernels with the steel plate. The end of each microphone was placed $25 \mathrm{~mm}$ from the point where kernels impacted the plate. Kernels were usually aligned lengthwise before dropping off of the end. However, during dropping, they often tumbled so their orientation was quite random when they striked the impact plate. This caused a great discrepancy in the amplitude of the acoustic signals emitted. Preliminary experiments showed that an order of magnitude difference 
in the intensity of the acoustic emissions among kernels was common. Thus, one microphone was amplified (2690 NEXAS, Bruel and Kjaer North America, Norcross, GA) at $1 \mathrm{~V} / \mathrm{Pa}$ and the other at $10 \mathrm{~V} / \mathrm{Pa}$ to ensure the system had the required dynamic range to capture the acoustic emissions. Microphone signals were digitized using a sound card (Waveterminal 192X, Ego Sys, Seoul, South Korea) at a sampling frequency of $192 \mathrm{kHz}$ with 16-bit resolution. This sound card does not have a $20 \mathrm{kHz}$ low pass filter that most consumer sound cards do use. The data acquisition was triggered using an optical sensor (QS30LLPC, Banner Engineering Corp. Minneapolis, MN). Upon receipt of a trigger signal, the computer acquired 2000 data points from the microphone signal. A high-pass filter was applied to the acquired signal using a single-pole recursive filter with a cutoff frequency of $9600 \mathrm{kHz}$. This filtering effectively eliminated $60 \mathrm{~Hz}$ noise, any DC offset, and eliminated effects from audible ambient sounds.

\subsection{Data processing}

Features from the $10 \mathrm{~V} / \mathrm{Pa}$-amplified microphone signal were extracted if less than six sampled points were saturated; otherwise, features extracted from the $1 \mathrm{~V} / \mathrm{Pa}$-amplified microphone signal were used. The signal processing and feature extraction were identical for each of the two amplified signals. Extracted features, along with the microphone gain, were presented to a neural network for classification. Four different methods were used for the feature extraction: (1) modeling of the signal in the time-domain; (2) computing timedomain signal variances in short-time windows; (3) analysis of the frequency spectrum magnitudes; and (4) differential spectral analysis. Discussion of each method follows.

\subsubsection{Time-domain signal modeling}

Typical signals from an undamaged kernel and IDK are shown in Fig. 4. The maximum amplitude of the signals are quite variable and are not useful for distinguishing IDK from undamaged kernels. However, IDK's have a consistent tendency to resonate for a longer time than undamaged kernels. This phenomenon is due to the insect tunnel within the body of the kernel. Similar acoustical response have been observed while grinding dif-
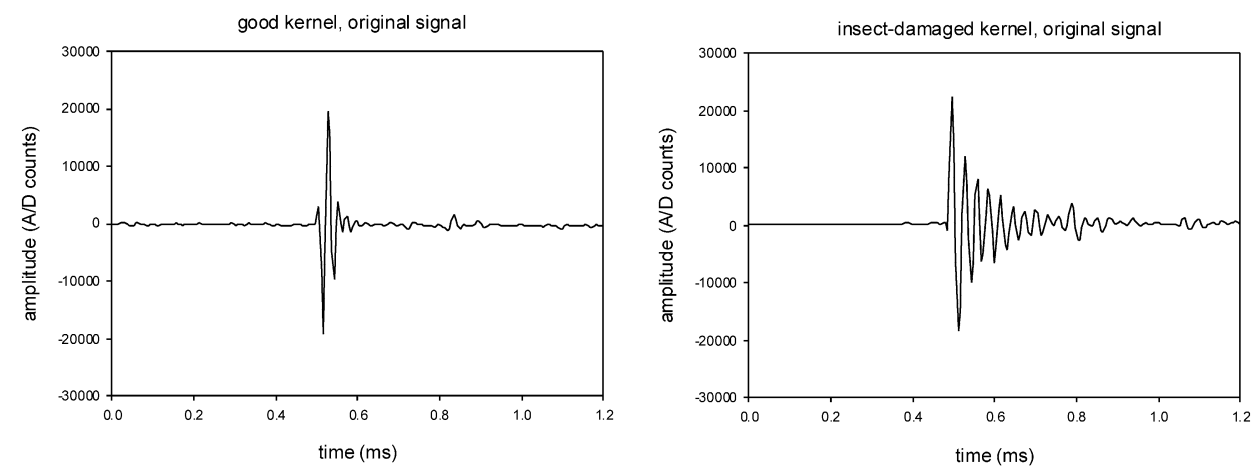

Fig. 4. Typical signals from an undamaged kernel (left) and IDK (right). Note that duration of the IDK signal is longer. 

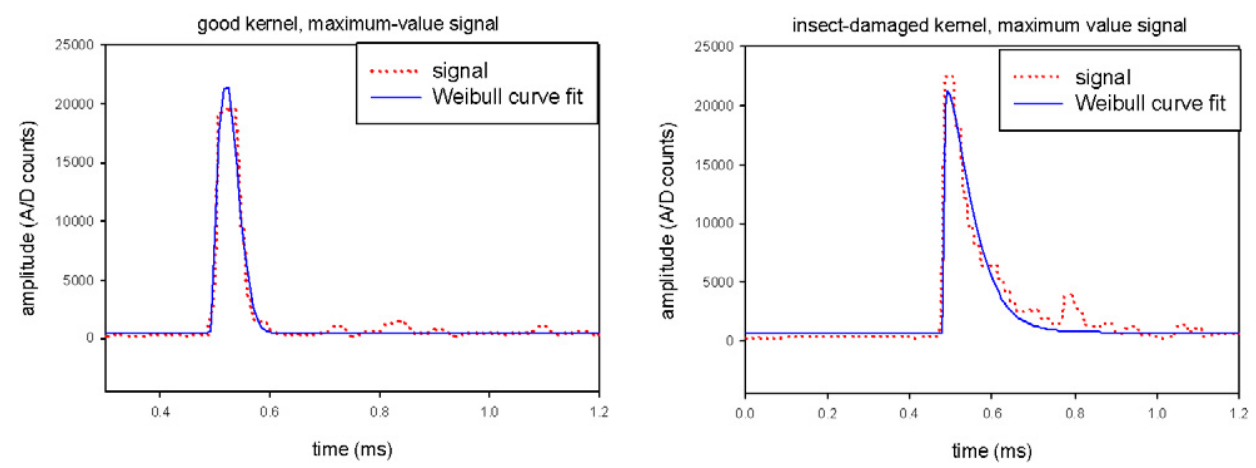

Fig. 5. Maximum-value filtered signals fitted with a Weibull curve for an undamaged kernel (left) and IDK (right).

ferent types of harder and softer wheat kernels [33]. Preliminary attempts to use damped sinusoids [26] to model the signals were not successful for distinguishing IDK and undamaged kernels. IDK acoustic emissions often, but not always, changes from an initially high frequency to a lower frequency before the signal diminishes. Thus, modeling with a damped sinusoid often showed that IDK signals diminished more rapidly than they actually did. Signal modeling was performed using the following three-step transformation: (1) rectifying the signal by taking the absolute value at all points; (2) non-linear filtering of the signal by replacing the center data point with the maximum value in a seven-point window in order to accentuate the peak in each wave period; and (3) non-linear estimation of the four parameters of the Weibull function, given by Eq. (1), which has a shape similar to that of the processed time-domain signal.

$$
Y=\frac{c b}{a}\left[\frac{\left(x-x_{0}\right)}{a}\right]^{(b-1)}\left\{e^{-\left[\frac{\left(x-x_{0}\right)}{a}\right]^{b}}\right\},
$$

where $x>x_{0}$, otherwise $Y=0$.

Parameters of the Weibull equation, $a, b, c$ and $x_{0}$, were iteratively estimated by a nonlinear regression dynamic-link library (DLL) (NLREG, Phil Sherod, Brentwood, TN). $Y$ is the magnitude of the Weibull function at data point $x$. Parameter $a$ controls the width of the curve while $b$ controls the shape; $c$ reflects the magnitude of the signal; and $x_{0}$ determines where the Weibull curve begins. For values of $b$ approaching 3.5, the curve approximates a Gaussian; when $b$ is near 2, the curve approximates a log-normal curve; and when $b$ approaches 1, the curve approximates an exponential decay. Thus, parameters $a$ and $b$ are potentially useful features for distinguishing IDK from undamaged kernels irregardless of the signal magnitude. Additionally, the proportion of variation explained by the model, $R^{2}$, can also aid in the identification of IDK. Figure 5 shows sample signals that have been processed as described above, and Table 1 shows average parameter estimates.

\subsubsection{Short-time variance window processing}

Variances and maximums of the signals were computed in short-time windows of the high-pass filtered time domain signal. While the Weibull function captures the shape of the recorded signal globally, the short-time variance information models the local time- 
Table 1

Average Weibull parameter estimates

\begin{tabular}{lllllr}
\hline Parameter & \multicolumn{2}{c}{ Undamaged } & & \multicolumn{2}{c}{ Insect-damaged (IDK) } \\
\cline { 2 - 3 } \cline { 6 - 6 } & Avg & Std dev & & Avg & Std dev \\
\hline$a$ & 15.01 & 8.06 & 27.99 & 19.37 \\
$b$ & 2.75 & 1.19 & 1.59 & 0.78 \\
$R^{2}$ & 0.85 & 0.17 & 0.80 & 0.13 \\
\hline
\end{tabular}

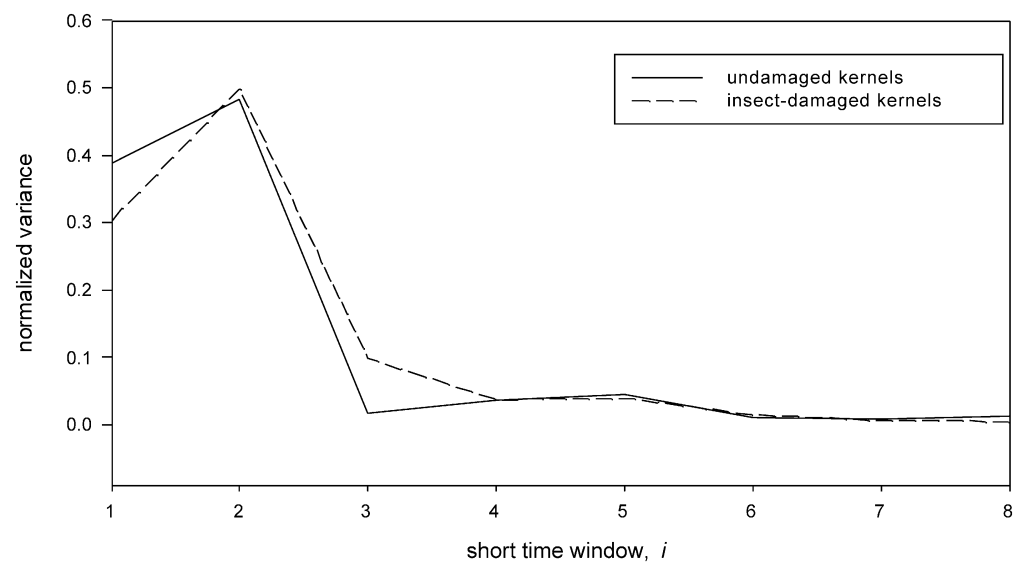

Fig. 6. Average normalized variances from short-time windows of time-domain signals.

domain variations in the signal. For variance computations, the short-time windows were 50 points in duration and incremented in steps of 30 points so that each window overlapped by 20 points. The first window began 40 points in front of the maximum-signal magnitude. A total of eight short-time windows were computed to cover the entire duration of all signals. After all variances were computed, they were normalized by the sum of all eight variances as described by the equation

$$
\sigma_{\mathrm{n} i}^{2}=\frac{\sigma_{i}^{2}}{\sum_{i=1}^{8} \sigma_{i}^{2}},
$$

where $\sigma_{\mathrm{n} i}^{2}$ and $\sigma_{i}^{2}$ are the normalized and computed variances from window $i$ with $i=1$ being the first window and $i=8$ being the last. This method captures the increased duration of the signals from insect-damaged kernels. Figure 6 shows the average normalized variance for IDK and undamaged kernels. IDK and undamaged kernels can be distinguished by the fact that the normalized variance of the third window is greater for IDK than for undamaged kernels, and the slope between the third and fourth normalized variance differs between the two.

For maximum-magnitude computations, the short-time window consisted of ten points with no overlap between the windows. Five windows were used, the first beginning at the maximum magnitude for the entire signal. As in Wiebull modeling, this computation relates to the duration of the signal ringing, but it also captures more localized variations than in 


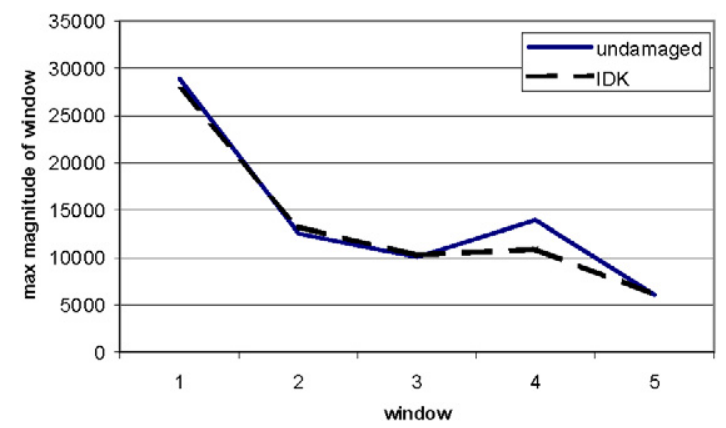

Fig. 7. Maximum rectified signal magnitudes of 10 point, short-time windows after the global signal maximum magnitude. Note the difference between IDK and undamaged kernels in the fourth window.

the Wiebul model. For this computation, the maximum magnitude of the rectified signal was computed and recorded, producing five points that describe the general magnitude of the signal in the 50 points immediately following the signal maximum magnitude. Figure 7 shows the average of these magnitudes for undamaged kernels and IDK. As found for the short-time variance windows, the values differ between the third and fourth windows, with the value of the magnitude increasing substantially for undamaged kernels. Otherwise, maximum magnitudes for undamaged and IDK are very similar.

\subsubsection{Frequency domain processing}

A 256-point DFT was computed from each signal using a Hamming window. The 256point window started 80 data points before the signal maximum slope, corresponding to the moment of impact, and covered the duration of the sound generated by the impact. The magnitude of each spectrum was computed, and a 20-tap FIR low-pass filter was applied to remove jagged spikes in the spectrum. The filter cutoff frequency was $\pi / 4$ in the normalized DFT domain. Figure 8 shows the frequency spectrum of an IDK, revealing a sharper peak at a lower frequency than for undamaged kernels. It should be noted that the DFT of the two kernels shown in Fig. 8 are obviously quite different but it emphasizes the major difference found in the DFT of IDK and undamaged kernels. Higher resolution DFTs have shown that IDK in fact have two peaks. However, this feature is often lost in noise. Peaks of the spectra of undamaged kernels and IDK are clearly distinguishable. However, there are significant overlaps in many cases, which are mainly due to the tumbling of the wheat kernel as it strikes the metal plate. The frequency corresponding to the peak magnitude in the frequency spectrum was saved as a potential discriminating feature. In addition, the 15 magnitude values before the peak and 15 points after the peak were saved and normalized to the peak magnitude. For undamaged kernels, the average frequency corresponding to the peak DFT magnitude was $34.5 \mathrm{kHz}$ with a standard deviation of $9.1 \mathrm{kHz}$. In contrast, IDK had an average peak frequency of $26.3 \mathrm{kHz}$ with a standard deviation of $9.6 \mathrm{kHz}$.

\subsubsection{Differential spectral analysis}

Preliminary testing using cepestral coefficients and mel-cepstral coefficients as they were applied to pistachio impact emissions by Cetin et al. [22] did not add any discrimi- 


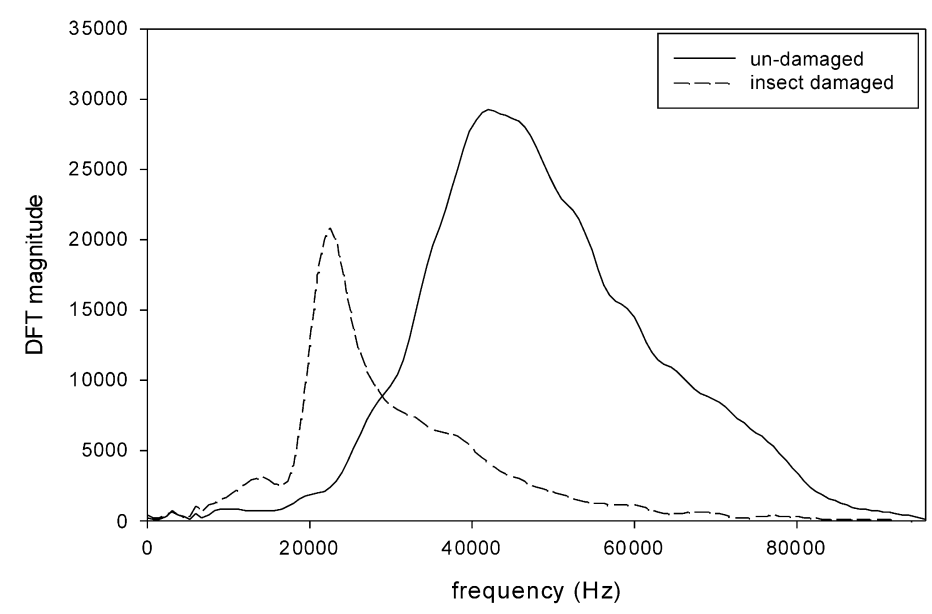

Fig. 8. Example frequency-spectrum magnitudes for an undamaged kernel and IDK.

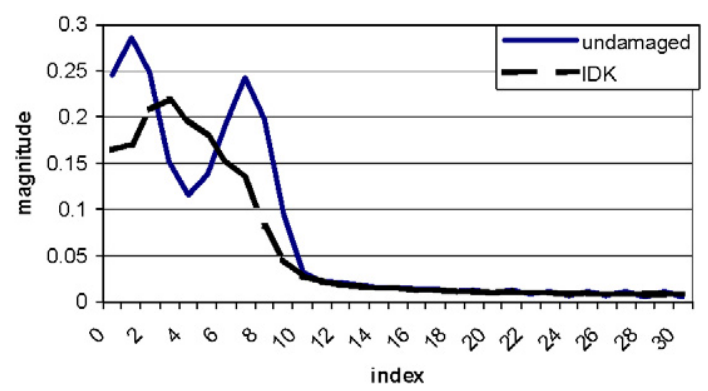

Fig. 9. Average magnitudes of the differential spectrum. Note the undamaged kernels have two distinct peaks compared with one for IDK, emphasizing the smaller oscillations observed in spectra from undamaged kernels of the time-domain signal.

nating power to the other features discussed thus far. As noted earlier, spectra from IDK had a tendency for one or two sharper peaks. In contrast, spectra from undamaged kernels tend to have one larger peak and many smaller oscillations between the frequencies of 33 and $81 \mathrm{kHz}$. Thus, the first derivative of the DFT was computed between these frequencies to emphasize these smaller oscillations and help deconvolute other peaks. This is a technique commonly used in near-infrared spectroscopy to extract peaks from smoothed data [34]. The derivative of the DFT magnitude, comprised of the 64 points between 33 and $81 \mathrm{kHz}$, was computed numerically using equation 3 followed by a 64-point DFT (on the resulting derivative spectrum) to produce 32 magnitude values of the differential spectrum. The magnitudes of this spectrum are shown in Fig. 9.

$$
S_{n}^{\prime}=S_{n-1}-S_{n+1},
$$

where $S_{n}$ is the magnitude of the spectrum at frequency index $n$. The computation was performed for all $n$ between 43 and 107, which corresponded to 33 and $81 \mathrm{kHz}$, respectively. 


\subsubsection{Classification}

The following features were pooled together as potential discriminating features: Weibull curve-fit parameters $a, b$, and $R^{2}$; normalized variances from the eight short-time windows; maximum values from five short-time windows; the frequency corresponding to the peak DFT magnitude; 30 normalized DFT magnitudes centered about the frequency corresponding to the peak DFT magnitude; the entire 128 DFT magnitudes; and the 32 magnitudes of the differential spectrum. Stepwise discriminant analysis (significance for entry and exit $=0.1$ ) was used to select a maximum of 20 of these features for classification by a neural network. This relatively low number of features was used to guard against the possibility of over-fitting the neural network. As an additional guard against over-fitting, the data sets were evenly divided into three randomly selected data sets henceforth called training, testing, and validation. Feature selection with stepwise discriminant analysis [35], followed by neural network training, was performed on the training set. Training was stopped when classification error difference between the training and test sets exceeded $3 \%$. The final trained neural network was applied to the validation set and these classification accuracies are reported. Two neural network calibrations were performed. One neural net was trained to discriminate IDK from undamaged kernels only. This network was then applied to all other damaged kernels (scab damage, sprouted, and kernels damaged by an insect at the pupae stage) to determine how other damaged kernels would be classified when presented to an IDK-detection neural net. The second neural network was trained to discriminate undamaged kernels from IDK plus a third group comprising scab, sprouted, and hidden insect-damaged kernels. Commercial neural network software was used (Alyuda Neurointelligence 2.1, Alyuda Research Corp., Los Altos, CA). The conjugate gradient algorithm [36] was used as the neural network training method. This method does not require specification of momentum or learning rate as back propagation methods do. The network architecture contained one hidden layer, and the number of hidden nodes was determined using an exhaustive search from one to 60 input nodes. As the number of features to be extracted has an impact on the processing time for real time sorting, the networks were tested using various combinations of the most significant features to determine how many features were required for maximum accuracy.

\section{Results}

\subsection{Discrimination between IDK and undamaged kernels (first network)}

The stepwise discriminant analysis procedure selected 19 features from the pool of all potential features. The six features selected as most significant were two magnitudes on each side of the peak of the DFT spectrum, the DFT magnitudes at 19.5, 2.25, and $40.5 \mathrm{kHz}$, and the fourth magnitude in the differential spectrum. The selection of the DFT magnitudes near the peak of the DFT spectrum as the two most significant features is not surprising as this characterizes the sharpness of the peak in the frequency spectrum. The selection of DFT magnitudes at $19.5,2.25$, and $40.5 \mathrm{kHz}$ simply show that there were significant differences in the general shape of the DFT between IDK and undamaged kernels. Undamaged kernels tended to have more energy at higher frequencies than IDK. Finally, 
Table 2

Validation set classification accuracies for subsets of features

\begin{tabular}{lll}
\hline Features & \multicolumn{2}{l}{ Percent accuracy } \\
\cline { 2 - 3 } & Undamaged & IDK \\
\hline Weibull $a, b$, and $R^{2}$ & 87 & 71 \\
Short-time window maximums & 88 & 72 \\
Short-time window variances and maximums & 86 & 76 \\
DFT peak area information & 92 & 85 \\
Complete DFT & 91 & 77 \\
Differential spectrum & 78 & 85 \\
Short-time window variances and maximums + DFT peak information & 94 & 86 \\
Top 6 selected features & 96 & 87 \\
19 selected parameters from all features & 98 & 89
\end{tabular}

Table 3

Classification of other types of damaged kernels by inputting the 19 features selected to distinguish IDK from undamaged kernels only to the neural network

\begin{tabular}{lll}
\hline Actual damage category & Percent accuracy \\
\cline { 2 - 3 } & Undamaged & IDK \\
\hline Scab & 96 & 4 \\
Sprout & 95 & 5 \\
Pupae & 95 & 5 \\
\hline
\end{tabular}

as pointed out earlier, the differential spectrum helps to identify smaller oscillations in the DFT. For undamaged kernels, whose DFT's tend to have a single broad peak accompanied by smaller oscillations, the differential spectra show one peak at a low frequency and another at a higher frequency (Fig. 9). In contrast, differential spectra from IDK tend to have one peak at a moderate frequency as DFT from IDK tend to have one or two sharp peaks. Thus, the stepwise discriminant procedure selected the fourth point, which tends to be the peak for IDK and a local minimum for undamaged kernels. The Neural network was found to perform best with 19 input nodes and 33 hidden nodes. Table 2 shows the classification accuracies obtained when various combinations of the selected features were input into the network.

The best results, $89 \%$ of IDK detected with $2 \%$ false positive, were obtained using all 19 features. Results using only the top six features as input vectors were only slightly worse, with $96 \%$ of IDK detected and $4 \%$ false positives. The number of features used in relation to the size and diversity of the training set indicates that over-fitting of the neural network was not likely. Results gradually degraded as fewer input vectors were used.

Table 3 shows the classification results for other types of damage when presented to the neural network trained to discriminate IDK from undamaged kernels. These results indicate other types of damage are unlikely to be classified as IDK using this neural network. A maximum of $6 \%$ of kernels with other types of damage were classified as IDK. While this may be desirable, depending on the sorting criteria, there is the disadvantage that this network was not able to detect hidden insect infestations. 
Table 4

Classification results for network trained to distinguish undamaged and IDK, plus a third group including all other damage

\begin{tabular}{lccc}
\hline Actual damage category & Percent accuracy & \\
\cline { 2 - 4 } & Undamaged & IDK & Scab, sprouted, or pupae \\
\hline Undamaged & 91 & 2 & 7 \\
IDK & 7 & 75 & 18 \\
Scab & 19 & 11 & 70 \\
Sprouted & 21 & 9 & 70 \\
Pupae & 33 & 22 & 45 \\
\hline
\end{tabular}

The classification results obtained for discriminating IDK from undamaged kernels compare favorably with X-ray imaging and NIR methods. Human examination of X-ray films is a more accurate method of detecting infested kernels at all maturity levels but can have false-positive errors of $1.0 \%$ or higher [8]. Computer algorithms to automatically scan X-ray images have similar recognition rates as the SKCS for insect-infested kernels but have higher false-positive rates, about 7.4\% [9]. NIR methods also suffer from false-positive errors and, additionally, kernel-orientation problems [37]. X-ray imaging equipment is very expensive, and software to segment images of wheat kernels that are touching each other leads to further errors. X-ray imaging likely would not be able to detect scab-damaged or sprouted kernels either. NIR can detect approximately $90 \%$ of IDK and scab-damaged kernels [6], with approximately $95 \%$ accuracy on undamaged kernels. Thus, the accuracy of NIR for damaged kernels is slightly higher at the expense of slightly higher false-positive errors. While NIR has been correlated with falling number tests with reasonable accuracy on bulk samples, no study has yet shown good accuracy with detecting sprout condition and alpha-amylase activity in single kernels. Thus, the acoustic method may have some additional usefulness in this application given the lack of another non-destructive method to detect sprout damaged kernels. NIR is more accurate than the acoustic method to detect kernels with insect pupae [17]. However, automated NIR instruments currently can have a throughput rate of approximately 1 kernel/s [38], while the acoustic method proposed here should be feasible at rates of 40 kernels/s or higher.

\subsection{Discrimination between undamaged, IDK, and other damage (second network)}

The stepwise discriminant procedure selected 20 features to a three way classification of kernels as undamaged, IDK, or having other damage (scab, sprout, pupae). As with IDK training, the two most significant features were two magnitudes near the peak of the DFT spectrum, followed by the DFT magnitude at $19.5 \mathrm{kHz}$, the fourth magnitude in the differential spectrum, the maximum value in the fourth short-time window, and the DFT magnitude at $67.5 \mathrm{kHz}$. Table 4 displays a confusion matrix of the classification results.

The results indicate that it is possible to distinguish undamaged kernels from IDK and kernels with other types of damage with reasonable accuracy for IDK, scab and sprouted kernels; but, poor accuracy for kernels infested with insects at the pupae level. For example, a very poor quality sample comprising 3000 kernels $(\sim 100 \mathrm{~g})$ might contain 30 IDK, 30 scab damaged, 30 sprouted, 30 pupae and the remaining 2880 kernels would be un- 
damaged. Thus, this hypothetical sample would comprise $96 \%$ undamaged kernels and $1 \%$ from each of the four damage types. A system could first classify kernels as IDK and divert those one way, then classify kernels as having "other damage" and divert those another direction, while undamaged kernels would go a third direction. This would produce an undamaged stream containing 2645 kernels, of which 99.1\% (2621 kernels) are undamaged, $0.08 \%$ (2 kernels) are IDK, $0.23 \%$ (6 kernels) are scab damaged, $0.23 \%$ (6 kernels) are sprouted, and $0.37 \%$ (10 kernels) have pupae damage. The "other damage" stream would comprise less than $10 \%$ of the original sample, 263 kernels, with $76.8 \%$ of these being undamaged, $2 \%$ being IDK, $8 \%$ being scab damaged, $8 \%$ being sprouted, and 5 having pupae damage. Given the potential high-throughput rate for an acoustic-based sorter, a large sample could be processed quickly and inspectors need only examine the reject streams. Using the algorithm presented here, the signal processing required $20 \mathrm{~ms} /$ kernel to perform, with the Weibull curve fitting taking about half this time. If Weibull features are not used, then computational time is less than $10 \mathrm{~ms} / \mathrm{kernel}$ on a Windows-based PC system using an AMD Athlon 3000 processor. If an air valve is used to divert damaged kernels, it will cause a disturbance of approximately $15 \mathrm{~ms}$ where acquiring data on a new kernel cannot be made. Thus, processing and removing kernels requires approximately $25 \mathrm{~ms}$ for a kernel throughput rate of 40 kernels/s.

\section{Conclusions and future work}

This work shows that use of impact-acoustic emissions as a basis for detection of IDK, sprout damage, and scab damage is a feasible and promising method. The computational cost of classifying a kernel using this technique is very low, allowing inspection of large numbers of wheat kernels very rapidly, $\sim 40$ kernels/s. Grain inspectors usually use a $100 \mathrm{~g}$ (3000 kernel) sample to inspect for IDK. This takes an inspector approximately $20 \mathrm{~min}$ to do manually but can be accomplished in about $75 \mathrm{~s}$ with an acoustic system.

Further experimentation and development is needed to expand this method, using a wider range of wheat varieties grown under different climatic conditions. Additionally, wheat is stored at a range of moisture contents, typically between 9 and $13 \%$, so data from a range of moisture contents will be needed to determine its effect. Moisture content from a bulk sample may need to be added to the neural network so that its influence can be accounted for. Fortunately, moisture content can be easily measured and entered. Quite likely, further signal processing and a more elaborate neural network will be needed to obtain high classification accuracies on more varied data. Additionally, more study is needed to improve accuracy on kernels infested with insects that have not yet emerged from the kernels.

\section{References}

[1] C.M. Christensen, R.A. Meronuck, Quality Maintenance in Stored Grains and Seeds, University of Minnesota Press, Minneapolis, MN, 1986.

[2] T. Herman, R.K. Bequette, P.J. McClusky, J.R. Pedersen, J.G. Ponte, J.P. Shroyer, Quality Oriented Marketing of Hard Winter Wheat, Cooperative Extension Service, Kansas State University, Manhattan, KS, 1995. 
[3] J. Pederson, Insects: Identification, damage, and detection, in: D.B. Sauer (Ed.), Storage of Cereal Grains and Their Products, fourth ed., American Association of Cereal Chemists, St. Paul, MN, 1992.

[4] USDA. Electronic code of federal regulations. Title 7 (Agriculture), Chapter VIII (Federal Grain Inspection Service), Part 810 (Official United States Standards for Grain), available at: www.usda.gov/gispa/ lawsandregs/lawsregs.htm, accessed 5 January 2004.

[5] FDA. Adulteration by insect and rodent filth. Compliance policy guide CPG7104.03, Section 578.300, available at: www.fda.gov/ora/compliance_ref/cpg/cpgfod/cpg578.300.htm, accessed 5 January 2004.

[6] G.E. Russell, Evaluation of four analytical methods to detect weevils in wheat: Granary weevil, Sitophilus granarius (L), in soft white wheat, J. Food Protect. 51 (7) (1988) 547-553.

[7] R.P. Haff, X-ray Inspection of Wheat for Granary Weevils, PhD dissertation, University of California, Davis, CA, 2001.

[8] R.P. Haff, Real-time X-ray inspection of wheat for infestation by the granary weevil, Sitophilus granarius (L), Trans. ASAE 47 (2) (2004) 531-537.

[9] C. Karunakkaran, D.S. Jayas, N.D.G. White, Soft X-ray inspection of wheat kernels infested by Sitophilus oryzae, Trans. ASAE 46 (3) (2003) 739-745.

[10] K.W. Vick, J.C. Webb, B.A. Weaver, C. Litzcow, Sound detection of stored-product insects that feed inside kernels of grain, J. Econ. Entomol. 81 (5) (1988) 1489-1493.

[11] R.W. Mankin, D. Shuman, J.A. Coffelt, Acoustic counting of adult insects with differing rates and intensities of sound production in stored wheat, J. Econ. Entomol. 90 (4) (1997) 1032-1038.

[12] D. Shuman, J.A. Coffelt, K.W. Vick, R.W. Mankin, Quantitative acoustical detection of larvae feeding inside kernels of grain, J. Econ. Entomol. 86 (3) (1993) 933-938.

[13] D.W. Hagstrum, Paul W. Flinn, Comparison of acoustical detection of several species of stored grain beetles (Coleoptera: Curculionidae, Tenebrionidae, Bostrichidae, Cucujidae) over a range of temperatures, J. Econ. Entomol. 86 (4) (1993) 1271-1278.

[14] W.A. Bruce, W.M. Street, R.C. Semper, D. Fulk, Detection of Hidden Insect Infestations in Wheat by Infrared Carbon Dioxide Gas Analysis, ARS bulletin AAT-S-26, July 1982.

[15] T.F. Schatzki, E.K. Wilson, G.B. Kitto, P. Behrens, I. Heller, Determination of hidden Sitophilus granarius (Coleoptera: Curculionidae) in wheat by myosin ELISA, J. Econ. Entomol. 86 (5) (1993) 1584-1589.

[16] E.B. Maghirang, F.E. Dowell, J.E. Baker, J.E. Throne, Automated detection of single wheat kernels containing live or dead insects using near-infrared reflectance spectroscopy, Trans. ASAE 46 (4) (2003) 1277-1282.

[17] F.E. Dowell, J.E. Throne, J.E. Baker, Automated nondestructive detection of internal insect infestation of wheat kernels by using near-infrared reflectance spectroscopy, J. Econ. Entomol. 91 (4) (1998) 899-904.

[18] T.C. Pearson, Automated detection of internal insect infestations in whole wheat kernels using a Perten SKCS 4100, Appl. Eng. Agricul. 19 (6) (2003) 727-733.

[19] S.R. Delwiche, Classification of scab- and other mold-damaged wheat kernels by near-infrared reflectance spectroscopy, Trans. ASAE 46 (3) (2003) 731-738.

[20] T.C. Pearson, Detection of pistachio nuts with closed shells using impact acoustics, Appl. Eng. Agricult. 17 (2) (2001) 249-253.

[21] A.E. Cetin, T.C. Pearson, A.H. Tewfik, Classification of closed- and open-shell pistachio nuts using voicerecognition technology, Trans. ASAE 47 (2) (2004).

[22] A.E. Cetin, T.C. Pearson, A.H. Tewfik, Classification of closed- and open-shell pistachio nuts using impact acoustical analysis, 2004, presented in ICASSP.

[23] D.B. Board, Incipient failure detection in high speed machinery, in: Proceedings of the 10th Symposium on NDE, San Antonio, 1975.

[24] V. Emamian, M. Kaveh, A.H. Tewfik, Z. Shi, L. Jacobs, J. Jarzynski, Robust clustering of acoustic emission signals using neural networks and signal subspace projections, EURASIP J. Appl. Signal Process. 2003 (3) (2003) 276-286.

[25] C. Chen, C. Mo, A method for intelligent fault diagnosis of rotating machinery, Digital Signal Process. 14 (2004) 203-217.

[26] T.J. Holroyd, Acoustics Emissions and Ultrasonic Monitoring Handbook, first ed., Coxmoor Publishing Company, Oxford, UK, 2000.

[27] B.B. Hubbard, in: A.K. Peters (Ed.), The World According to Wavelets: The Story of a Mathematical Technique in the Making, second ed., Natick, MA, 1998. 
[28] J.W. Tukey, B.P. Bogert, M.J.R. Healy, in: M. Rosenblatt (Ed.), The frequency analysis of time series for echoes: Cepstrum, pseudo-autocovariance, cross-cepstrum, and saphe-cracking, in: Proceedings of the Symposium on Time Series Analysis, Wiley, New York, 1963, pp. 209-243.

[29] F. Duprat, M. Grotte, E. Pietri, D. Loonis, The acoustic impulse-response-method for measuring the overall firmness of fruit, J. Agricult. Eng. Research 66 (4) (1997) 251-259.

[30] M.L. Stone, P.R. Armstrong, D.D. Chen, G.H. Brusewitz, N.O. Maness, Peach firmness prediction by multiple location impulse testing, Trans. ASAE 41 (1) (1998) 115-119.

[31] F.L. Younce, D.C. Davis, A dynamic sensor for cherry firmness, Trans. ASAE 38 (5) (1995) 1467-1476.

[32] J. Sugiyama, T. Katsurai, J. Hong, H. Koyama, K. Mikuriya, Melon ripeness monitoring by a portable firmness tester, Trans. ASAE 41 (1) (1998) 121-127.

[33] D. Massie, D. Slaughter, J. Abbott, W. Hruschka, Acoustic, single-kernel wheat hardness, Trans. ASAE 36 (5) (1993) 1393-1398.

[34] W.R. Hruschka, Multivariate calibration and data compression, in: P. Williams, K. Norris (Eds.), NearInfrared Technology in the Agricultural and Food Industries, first ed., American Association of Cereal Chemists, St. Paul, MN, 1987.

[35] C.J. Huberty, Applied Discriminant Analysis, Wiley, New York, 1994.

[36] C.M. Bishop, Neural Networks for Pattern Recognition, Clarendon Press, Oxford, MA, 1995.

[37] A.R. Ghaedian, R.L. Wehling, Discrimination of sound and granary weevil-larva-infested wheat kernels by near-infrared diffuse reflectance spectroscopy, J. AOAC Internat. 80 (5) (1997) 997-1005.

[38] F.E. Dowell, E.B. Maghirang, Accuracy and feasibility of measuring characteristics of single kernels using near-infrared spectroscopy, in: Proceedings of the ICC Conference 2002 "Novel Raw Materials, Technologies, and Products - New Challenge for Quality Control,” 2002, Budapest, pp. 313-320.

Tom C. Pearson received his PhD in engineering from University of California, Davis, in 1998. He is currently a lead scientist/engineer at the United States Department of Agriculture-Agricultural Research Service in Manhattan, Kansas. He is also an adjunct faculty at Kansas State University, Department of Biological and Agricultural Engineering. His current research interests are in the development of instrumentation and signal processing methods for rapid inspection and sorting of agricultural products. Specific applications include use of imaging, acoustics and near infrared spectroscopy for detecting defects in grains.

A. Enis Cetin received his $\mathrm{PhD}$ in systems engineering in 1987 from the Moore School of Electrical Engineering at University of Pennsylvania in Philadelphia. He is currently a Professor in Electrical and Electronics Engineering at Bilkent University in Ankara, Turkey. His current research interests are in the development of robust voice recognition systems in the presence of noise, motion detection and tracking in video streams for security systems, and applications of digital signal processing for improved inspection accuracy of agricultural products.

Ahmed H. Tewfik received his $\mathrm{PhD}$ in electrical engineering and computer science from the Massachusetts Institute of Technology in 1987. He is currently the E.F. Johnson Professor of Electronic Communications, Department of Electrical Engineering, University of Minnesota in Minneapolis. His current research interests include genomics, datanomic computing, wireless personal area communications, and food inspection.

Ron P. Haff received his PhD in engineering from University of California, Davis, in 2001. He is currently a lead scientist/engineer at the United States Department of Agriculture-Agricultural Research Service in Albany California. His current research interests are in the development of instrumentation and signal processing methods for rapid inspection and sorting of agricultural products. Specific methods include X-ray imaging and physical methods for detecting defects in fruits, nuts, and grains. 